\title{
Communication \\ Surface-Integrated Electric Field Sensor for the Detection of High-Voltage Power Lines
}

\author{
Gunbok Lee ${ }^{1}$, Jeong-Yeon Kim ${ }^{2}$, Gildong Kim ${ }^{1}$ and Jae Hee Kim ${ }^{2, *(1)}$ \\ 1 Propulsion System Research Department, Korea Railroad Research Institute (KRRI), Uiwang 16105, Korea; \\ gunbok@krri.re.kr (G.L.); gdkim@krri.re.kr (G.K.) \\ 2 School of Electrical, Electronics and Communication Engineering, Korea University of Technology and \\ Education, Cheonan 31253, Korea; jeongyeonday@koreatech.ac.kr \\ * Correspondence: jaehee@koreatech.ac.kr
}

Citation: Lee, G.; Kim, J.-Y.; Kim, G.; Kim, J.H. Surface-Integrated Electric Field Sensor for the Detection of High-Voltage Power Lines. Sensors 2021, 21, 8327. https://doi.org/ $10.3390 / \mathrm{s} 21248327$

Academic Editor: Yang Yue

Received: 2 November 2021

Accepted: 10 December 2021

Published: 13 December 2021

Publisher's Note: MDPI stays neutral with regard to jurisdictional claims in published maps and institutional affiliations.

Copyright: (C) 2021 by the authors Licensee MDPI, Basel, Switzerland. This article is an open access article distributed under the terms and conditions of the Creative Commons Attribution (CC BY) license (https:// creativecommons.org/licenses/by/ $4.0 /)$.

\begin{abstract}
When a drone is used for inspection of facilities, there are often cases in which high-voltage power lines interfere, resulting in the drone being caught or falling. To prevent this type of incident, drones must be capable of detecting high-voltage power lines. Typically, a strong electric field is formed around the high-voltage lines. To detect the electric fields around high-voltage lines, this study proposes an electric field sensor that may be integrated within the body of a drone. In a laboratory environment, a voltage of $25 \mathrm{kV}$ was applied to an overhead line, and the induced voltage in the proposed sensor was measured at various electric field intensities. Over an electric field range of 0.5 to $10.1 \mathrm{kV} / \mathrm{m}$, a voltage of 0 to $0.77 \mathrm{~V}$ was measured with each proposed sensor. In addition, the electric field and the voltage induced in the sensor were measured in a real-world railway environment with overhead lines. Under these conditions, the proposed sensor has the compensated value of 4.5 when the measured electric field was $4.05 \mathrm{kV} / \mathrm{m}$. Therefore, the proposed sensor may be applied in drones to measure large electric fields and to detect the presence of high-voltage lines in its vicinity.
\end{abstract}

Keywords: electric field sensor; drone; overhead line

\section{Introduction}

Recently, more and more drones are used for the inspection of electrical facilities. However, when these drones approach a facility to obtain high-resolution photos, the distances between the power lines and drone are not accurately identified, which may lead to the drone becoming caught in the lines during maneuvers. If the drone becomes caught in a high-voltage power line, the drone may be destroyed; furthermore, the high-voltage lines may be damaged, thereby affecting numerous electrical facilities connected to these high-voltage lines. Therefore, a method for detecting power lines is required when drones are used in the vicinity of high-voltage power lines.

A commonly used method for the detection of high-voltage lines utilizes images or videos taken during the drone operation. This method acquires image data near the drone and extracts information involving the voltage lines using algorithms such as the Hough transform, Radon transform, and Line Segment Detector [1,2]. By extracting this power line information using this method, the presence of power lines near the drone can be detected. However, when detecting these lines using the captured images, it is difficult to determine the exact locations and distances of the lines.

Several previous studies have proposed the measurement of the voltage of highvoltage lines via non-contact methods [3-6]. However, these methods do not provide information on the location of these high-voltage lines. To obtain voltage measurements for high-voltage lines, the electric field was measured at a specific location and the measured electric field values were converted into voltages. For measurement of the electric field, a 
D-dot E-field sensor with a parallel distributed electrode was developed, and sensor arrays were constructed to measure the voltage.

In the case of high-speed railways, power at $60 \mathrm{~Hz}$ and $25 \mathrm{kV}$ is supplied to the overhead line. When such a high voltage is applied to the overhead line, a strong electric field is generated around the overhead line. In this study, we propose a method for measuring electric fields such that drones may be able to detect high-voltage lines in their vicinity. Upon measuring an electric field, the presence of high-voltage lines in the surrounding area may be detected using the relative intensity of the measured electric field. Several methods have been proposed to obtain electric field measurement [7-12]. The most common method involves the use of a dipole antenna [8]. When a dipole antenna is employed, the varying electric field is detected by the two wires of the dipole antenna. The intensity of the electric field can be estimated by determining the voltage applied across the two wires. However, dipole antenna may only be reduced to a certain size because the magnitude of the detected signals is reduced as the size of the antenna is reduced. If the antenna is small, considerable amounts of noise are included, leading to increased errors in the measured electric field. It has been reported that, for a small dipole antenna, the signal-to-noise ratio (SNR) of the electric field was decreased by four times the scaling factor when the SNR was measured with different antenna lengths [10]. Therefore, it is advantageous to increase the size of the antenna when designing an antenna for measuring electric fields. Recently, a field mill sensor has been proposed to precisely measure the electric field in a high-voltage direct-current transmission line [12]. The measurement results in an actual high-voltage environment using the sensor were presented, and the results were also quite accurate. However, since the size of the proposed sensor is large, it is not a suitable application for drones.

In this study, we proposed a method involving the use of a component of a drone to measure the electric field generated by high-voltage power lines. If the body of the drone is used as an electric field sensor, the size of the sensor can be made large; thus, it has the advantage of increasing the induced voltage of the sensor. Moreover, there is no need to reserve additional space for the electric field sensor. If the electric field is constantly monitored, the presence of high-voltage lines in the vicinity of the drone can be inferred when the measured electric field is higher than a set threshold value. The drone may then avoid high-voltage lines based on the measured electric field values. The remainder of this paper is structured as follows. Section 2 examines the characteristics of general sensors and proposes a structure for the desired sensor such that it can be applied to drones. Section 3 verifies the operation of the sensor under strong electric fields using laboratory and field experiments. Finally, Section 4 outlines the findings and presents the conclusions of this study.

\section{Electric Field Sensor}

\subsection{Principles of Electric Field Measurements}

Electric fields and induced voltages are closely related. The structures most commonly applied for sensors used to measure electric fields are small dipole antenna. In addition, two parallel plates separated by a set distance can also be used as sensors for electric field measurements, although this type of the sensor is not commonly used. Figure 1 shows the structure of a small dipole antenna and that of two parallel plates. In general, when a small dipole is placed where an electric field is generated, the voltage induced in the small dipole can be expressed by the following equation $[9,13]$ :

$$
V_{O C}(\omega)=h E(\omega)
$$

where $V_{O C}$ is the voltage, $E(\omega)$ is the electric field, $\omega$ is the angular frequency, and $h$ is half of the total length of the small dipole antenna. 


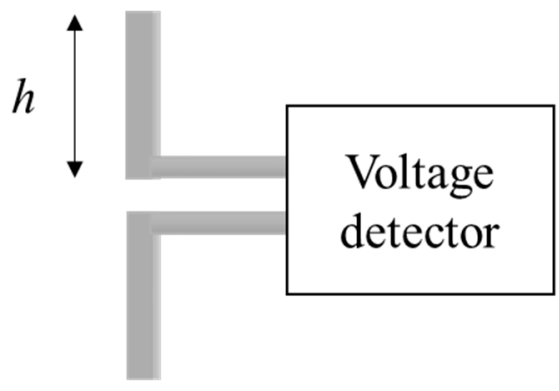

(a)

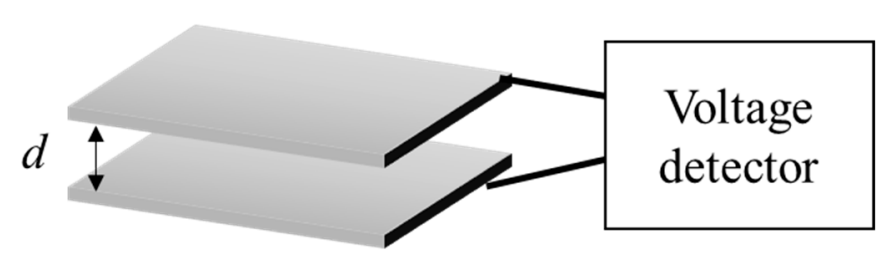

(b)

Figure 1. Conventional electric field sensors: (a) small dipole; (b) parallel plate capacitor.

Similarly, in the case of an electric field sensor composed of two parallel plates, the electric field formed between the parallel plates is directly related to the voltage applied between the two parallel plates. If the distance between the parallel plates is $\mathrm{d}$ and the magnitude of the electric field formed between the two parallel plates is $E(\omega)$, then the following relationship holds for the voltage $V_{O C}[14]$ :

$$
V_{O C}(\omega)=-E(\omega) d
$$

Assuming that the electric field is constant from Equations (1) and (2), it can be seen that the induced voltage increases when the dipole antenna is lengthened or the distance between two parallel plates is increased.

\subsection{Induced Voltage with Respect to the Direction of the Electric Field Sensor}

When a dipole and a parallel plate capacitor are used at a location with a uniform distribution of an electric field, the magnitude of the induced voltage varies depending on the orientation of the sensors. For the following simulations, the orientation of the sensors was varied, as shown in Figure 2, in a uniform electric field. To simulate the abovementioned configurations, a structure that creates a uniform electric field is required. Therefore, an electric field generator was constructed to generate an electric field of a constant magnitude in a uniform direction. The structure of the electric field generator used two large metal plates, and a voltage was applied. When a voltage is applied to the two plates, a uniform electric field is formed between the plates. Figure 3 shows the structure of the electric field generator and the orientation of the sensor in the simulation.

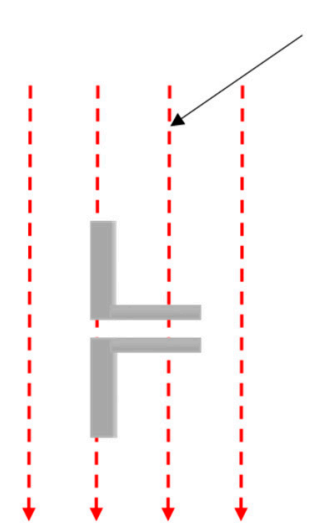

(a)

\section{Electric field distribution}

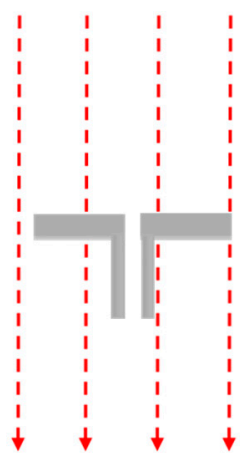

(b)

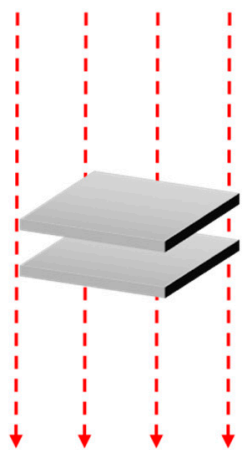

(c)

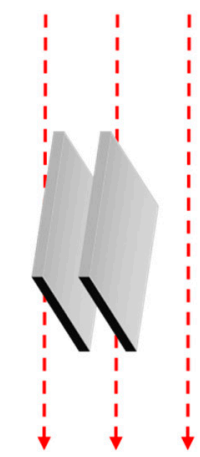

(d)

Figure 2. Orientation for the measurement of voltage induced by an electric field: (a) dipole (vertical); (b) dipole (horizontal); (c) parallel plate capacitor (horizontal); (d) parallel plate capacitor (vertical). 


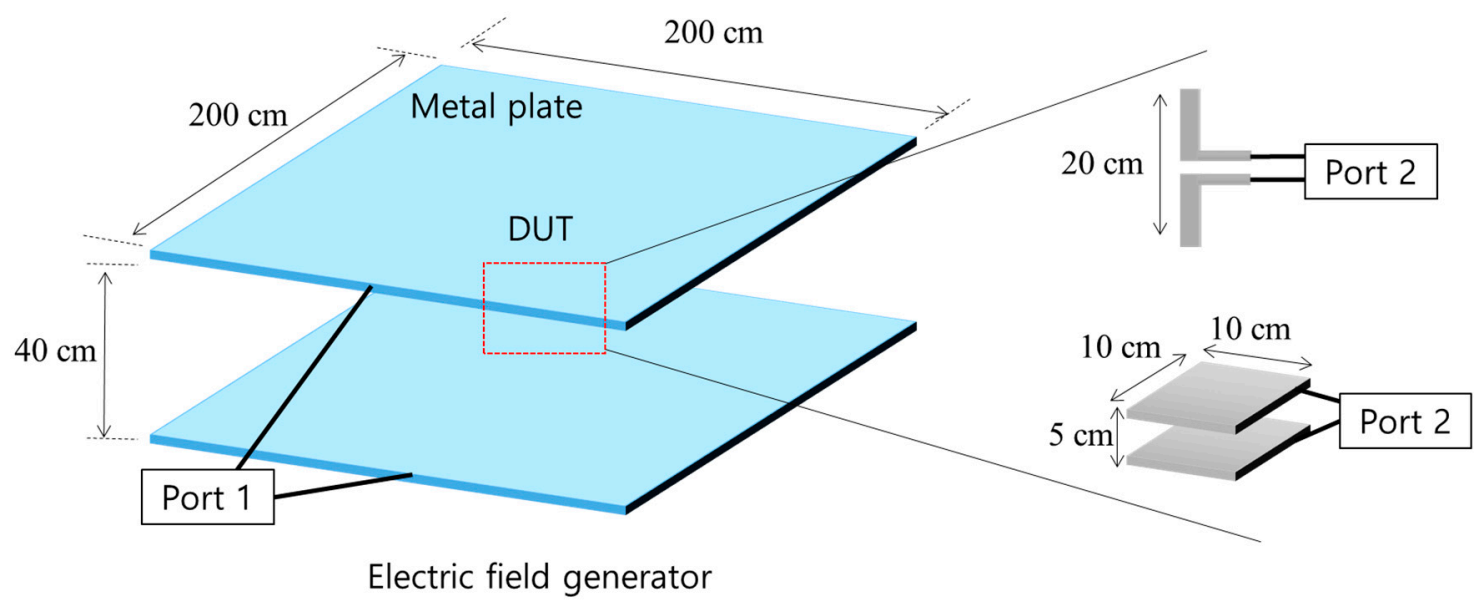

Figure 3. Schematic of the structure used for the simulation of the induced voltage with respect to the orientation of the sensor.

The horizontal and vertical lengths of the plate were $200 \mathrm{~cm}$, and the distance between the two plates was set to $40 \mathrm{~cm}$. The voltage was applied through port 1 , and $1000 \mathrm{~V}$ were applied in the simulation. Although the frequency of a real-world overhead power line voltage is $60 \mathrm{~Hz}$, it is difficult to observe clear characteristics under these conditions via simulation because the induced voltage in the sensor is low at frequency of $60 \mathrm{~Hz}$. Because the induced voltage in the sensor is generally proportional to the frequency, a frequency of $60 \mathrm{kHz}$ (1000 times greater than the conventional power line frequency) was used in the simulations. The increase in induced voltage according to the increase in frequency can be derived from the equivalent circuit model. For the structures of Figure 1, the equivalent circuit model can be represented as Figure 4. [13]. The induced voltage of the load impedance $Z_{L}(\omega)$ is expressed by the following equation.

$$
V_{L}(\omega)=V_{O C}(\omega) \frac{Z_{L}(\omega)}{Z_{A}(\omega)+Z_{L}(\omega)}
$$

where $Z_{A}(\omega)$ is the input impedance of the sensors. For the small dipole in Figure 1a, the sensor impedance $Z_{A}(\omega)$ can be expressed as follows.

$$
Z_{A}(\omega)=-j \frac{\eta_{0}}{2 \pi \beta h}(\Omega-2-\ln 4)
$$

where $\eta_{0}$ is free space wave impedance $(120 \pi \mathrm{ohms})$ and $\Omega$ is thickness factor. $\Omega=$ $2 \ln (2 \mathrm{~h} / \mathrm{a})$. where $\mathrm{a}$ is the antenna radius and $\mathrm{h}$ is the half length of the dipole antenna. $\beta=\omega \sqrt{\mu \varepsilon}$ is the propagation constant. From the Equation (4), If the frequency is high, $Z_{A}(\omega)$ becomes small. Therefore, the voltage induced by the sensor increases with higher frequency. For the parallel plate capacitor in Figure 1b, the sensor impedance can be simply regarded as the impedance of capacitor. If we assume the capacitance of the structure is $C_{P}$, the sensor impedance expressed by the following equation.

$$
Z_{A}(\omega)=-j \frac{1}{\omega C_{P}}
$$

From the Equation (5), as the frequency increases, the $Z_{A}(\omega)$ decreases; thus, the voltage induced in the sensor increases. 


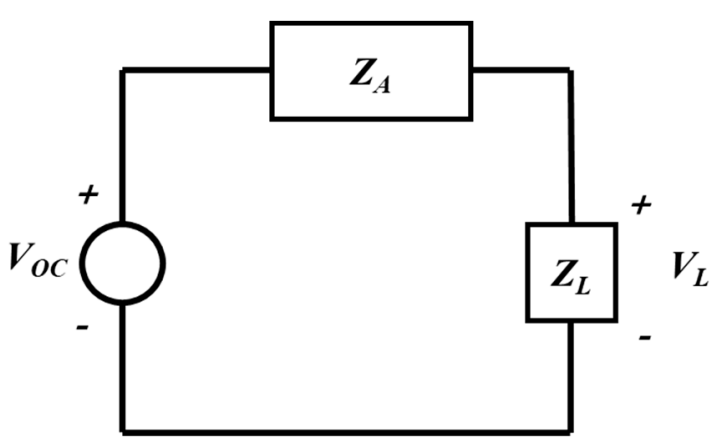

Figure 4. Equivalent circuit model of the conventional electric field sensors.

Considering the device under test (DUT), a small dipole antenna and parallel palate capacitor were used, and these were placed in the middle of the electric field generator. The length of the small dipole was set to $20 \mathrm{~cm}$, and the dimensions of the parallel plate capacitor were $20 \mathrm{~cm}$ in width and length and $5 \mathrm{~cm}$ in height. Next, each sensor was connected to port 2 with a load resistance of $1 \mathrm{M} \Omega$ to simulate the induced voltage. The simulations were performed using CST Studio Suite, which is a commercial software package for electromagnetic (EM) analysis. The results of the simulations are presented in Table 1.

Table 1. Induced voltage for the different orientations of the sensors.

\begin{tabular}{|c|c|c|}
\hline \multicolumn{2}{|c|}{ Sensors } & \multirow{2}{*}{$\frac{\text { Measured Voltage (V) }}{127}$} \\
\hline & Vertical & \\
\hline smant aspore antenta & Horizontal & 0.4 \\
\hline \multirow{2}{*}{ Parallel palate capacitor } & Vertical & 1 \\
\hline & Horizontal & 108 \\
\hline
\end{tabular}

By examining the voltages of the sensors induced in the simulations, it can be seen that, in the small dipole antenna, a voltage of $127 \mathrm{~V}$ is induced in the vertical orientation, while for the parallel plate capacitor, a voltage of $108 \mathrm{~V}$ is induced in the horizontal orientation. No voltage was induced in the horizontally placed dipole antenna or the vertically placed parallel plate capacitor. Therefore, it can be observed that the small dipole antenna and parallel plate capacitor have structures that detect electric fields that are generated in specific orientations.

\subsection{The Proposed Electric Field Sensor}

The structure of the sensor for measuring the relative intensity of an electric field is illustrated in Figure 5. Because drone-like structures are not long in the vertical direction, dipole antenna cannot be applied to z-axis. Moreover, it is difficult to make a sufficient induced voltage using only parallel plate capacitor because the height is low. To utilize the body of the drone as much as possible for the sensor, a copper plate of $5 \mathrm{~cm}$ in width and length was applied to the upper surface of the drone. A metal post was attached to one side of the drone's leg. The length of the metal attached to the leg of the drone was $11 \mathrm{~cm}$. The use of the leg of the drone as a part of the sensor structure is advantageous for receiving z-axis electric fields. A dipole antenna structure was used to measure the electric field in the $x$-axis and $y$-axis directions. Metal bars with a length of $9 \mathrm{~cm}$ and a width of $2 \mathrm{~cm}$ were attached to the four frames of the drone. A resistor of $1 \mathrm{M} \Omega$ was used to load impedance. This resistance serves to prevent sudden surges in the voltage measurements due to noise generated by the substrate and air. The relative intensity of the electric field can be determined by measuring the voltage across each sensor structures. The analog voltage is converted into a digital voltage using an analog digital converter (ADC), and the final value of the digitally converted signal determined via digital signal processing in 
the embedded board. A 16 bit ADS1115 was used as the ADC, and analog signal inputs from 0 to $4.09 \mathrm{~V}$ were converted into 16 bit digital values. Since a $60 \mathrm{~Hz}$ voltage source is used in the power line, the frequency of the electric field was also $60 \mathrm{~Hz}$. Therefore, the data sampling frequency must be higher than $120 \mathrm{~Hz}$ (two times $60 \mathrm{~Hz}$ ) according to the Nyquist sampling theory. The sampling rate of this system was $475 \mathrm{~s}^{-1}$. A Raspberry Pi 4.0 board was used as an embedded board for ease of data processing.
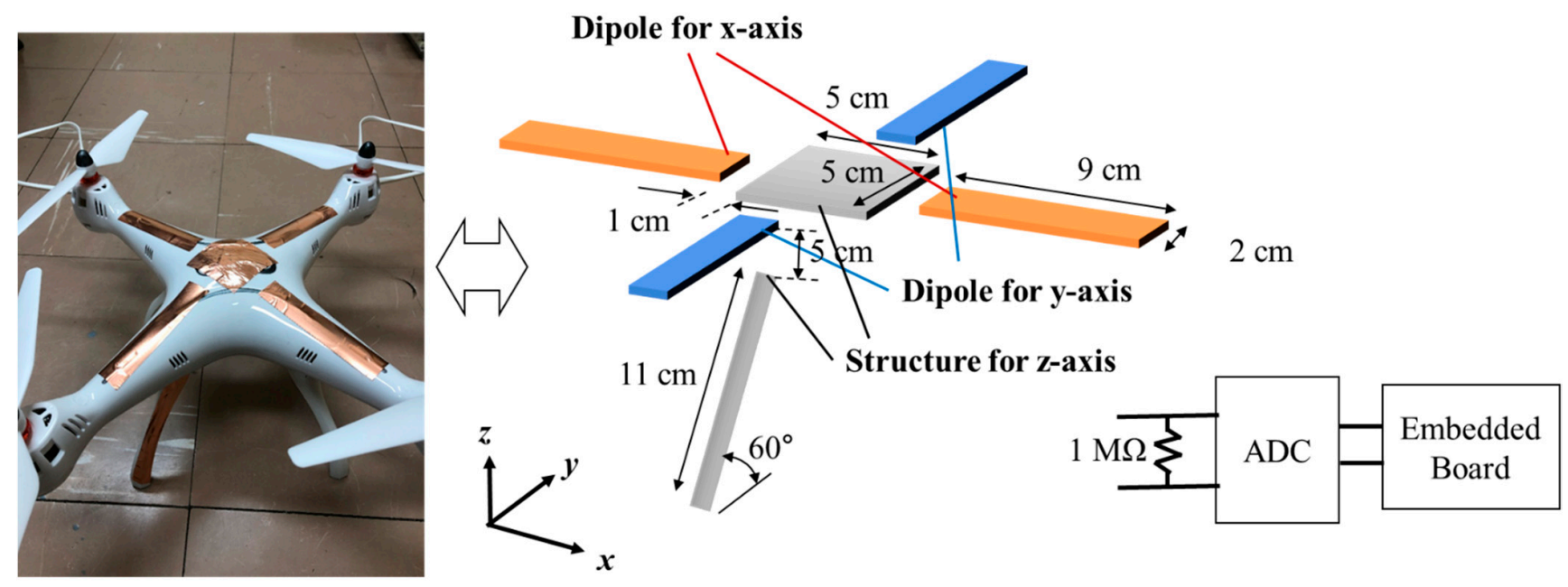

Figure 5. Photo and schematic of the structure of the proposed electric field sensor.

The induced voltages for the different orientations of the proposed sensor were simulated. The simulation environment was the same as in Section 2.2. The simulated result is shown in Table 2. 'Dipole for $\mathrm{x}$-axis' is a device for measuring the voltage induced from two metals arranged in the $x$ direction. 'Dipole for $y$-axis' refers to the structure of a dipole antenna arranged parallel to the y-axis. 'Structure for z-axis' is a structure for measuring the voltage induced between the upper metal plate and the drone's legs. From the simulation results, the proposed sensor well receives the electric field formed in each direction. However, in the case of the 'structure for z-axis', the drone's leg is curved in the $y$-axis direction, such that it receives electric fields in both the $y$-axis and $z$-axis directions.

Table 2. Induced voltage for the proposed sensors.

\begin{tabular}{ccc}
\hline Direction of E-Field & Proposed Sensors & Simulated Sensor Voltage (V) \\
\hline \multirow{2}{*}{ x-axis } & Dipole for x-axis & 177 \\
\cline { 2 - 3 } & Dipole for y-axis & 0.5 \\
\cline { 2 - 3 } & Structure for z-axis & 0.5 \\
\cline { 2 - 3 } y-axis & Dipole for x-axis & 0.7 \\
\cline { 2 - 3 } & Dipole for y-axis & 180 \\
\cline { 2 - 3 } & Structure for z-axis & 114 \\
\hline \multirow{2}{*}{ z-axis } & Dipole for x-axis & 4.5 \\
\hline & Dipole for y-axis & 8.5 \\
\cline { 2 - 3 } & Structure for z-axis & 111 \\
\hline
\end{tabular}

In the case of the dipole antenna, it is obvious that the induced voltage decreases as the length becomes shorter. Since the structure for z-axis uses upper metal plates and metal posts, it is necessary to investigate how the induced voltage changes according to the length change in this structure. The induced voltage of the sensor according to the length change is shown in Table 3. For the metal post, which is integrated with the drone's leg, the 
induced voltage decreases at a constant rate as the length of the metal post decreases with respect to the electric field on the z-axis and y-axis, respectively. In the case of the upper metal plate, the induced voltage increases as the size increases. In addition, more voltage is induced by the z-axis electric field than by the y-axis electric field. However, since the body size of the drone is fixed, there is a limit to increasing the size of the upper metal plate, and it is necessary to appropriately select the size of the plate in consideration of the size of the drone.

Table 3. Induced voltage for different size of structure for z-axis.

\begin{tabular}{cccr}
\hline \multirow{2}{*}{$\begin{array}{c}\text { Metal Length of Drone's Leg } \\
(\mathbf{c m})\end{array}$} & $\begin{array}{c}\text { Width and Length of Upper Metal } \\
\text { Plate }(\mathbf{c m})\end{array}$ & \multicolumn{2}{c}{ Simulated z-axis Sensor Voltage (V) } \\
\cline { 3 - 4 } & $2 \times 2$ & for y-axis E-Field & for z-axis E-Field \\
\hline 11 & $5 \times 5$ & 114 & 84 \\
\hline 11 & $8 \times 8$ & 123 & 137 \\
\hline 11 & $5 \times 5$ & 92 & 92 \\
\hline 8 & $5 \times 5$ & 75 & 74 \\
\hline 5 & & & 737 \\
\hline
\end{tabular}

\section{Results}

To measure the voltage induced in the sensor with respect to the electric field, an experimental setup was constructed, as shown in Figure 6. This setup reproduced a railway environment with overhead lines. Figure 6a shows the overall configuration of the experimental setup, which consisted of a ground plane, overhead line, and voltage source. Typically, a high voltage of $60 \mathrm{~Hz}$ and $25 \mathrm{kV}$ is supplied in the overhead lines of railway environments. Therefore, a voltage source of $60 \mathrm{~Hz}$ was used. In the experiment, the generated voltage was in the range of 1 to $25 \mathrm{kV}$. For the ground plane, a wide metal plate was used. The wide metal plate was connected to the ground of the voltage source, and the (+) terminal of the voltage source was connected to the overhead line. The overhead line was located $1.65 \mathrm{~m}$ above the ground plane. The length of the overhead line was $2 \mathrm{~m}$. The ground plane and overhead lines were electrically disconnected. When a voltage was applied to the overhead line, an electric field was generated between the overhead line and ground plane. To examine the effects of the electric field on the proposed sensor, the proposed sensor (DUT) and a commercial electric field analyzer were placed between the overhead line and ground plane. An electric and magnetic field analyzer (EHP-50F) was used to measure the $60 \mathrm{~Hz}$ electric field in the vicinity of the overhead line. Data were acquired at various positions, and the details are presented in Figure 6c. Because the electric field is a vector quantity, it has a direction. The $\mathrm{x}, \mathrm{y}$, and $\mathrm{z}$ directions of the field analyzer are indicated in Figure $6 \mathrm{~b}$. 


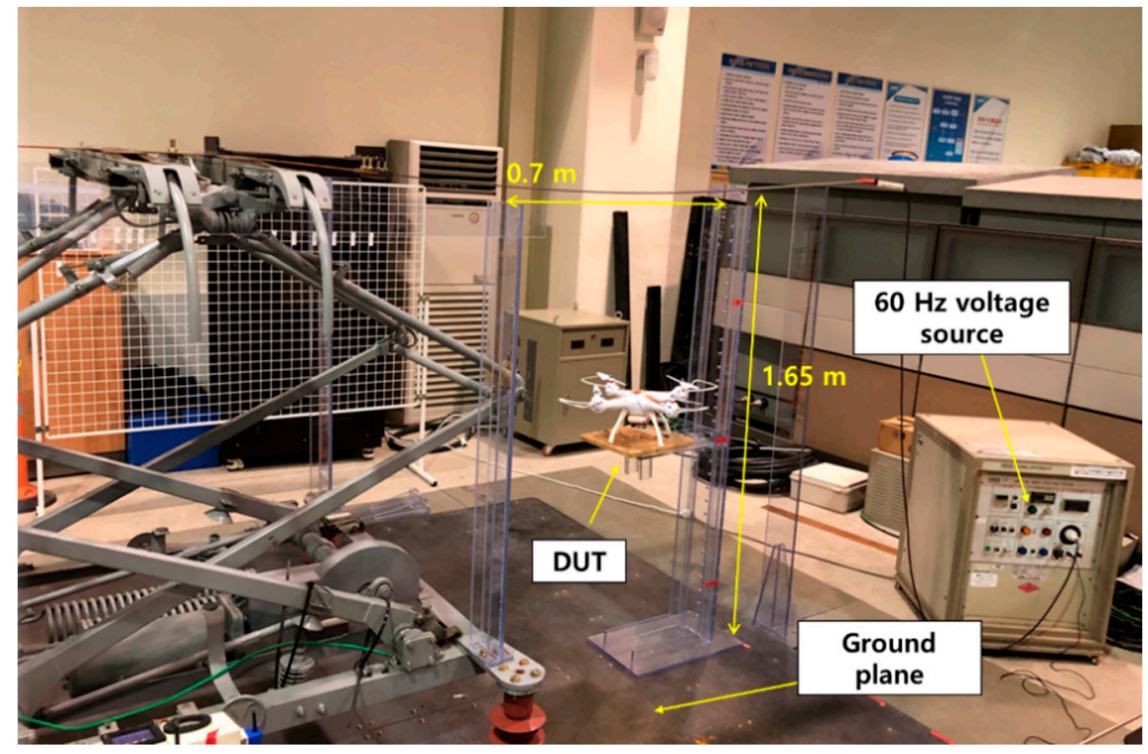

(a)
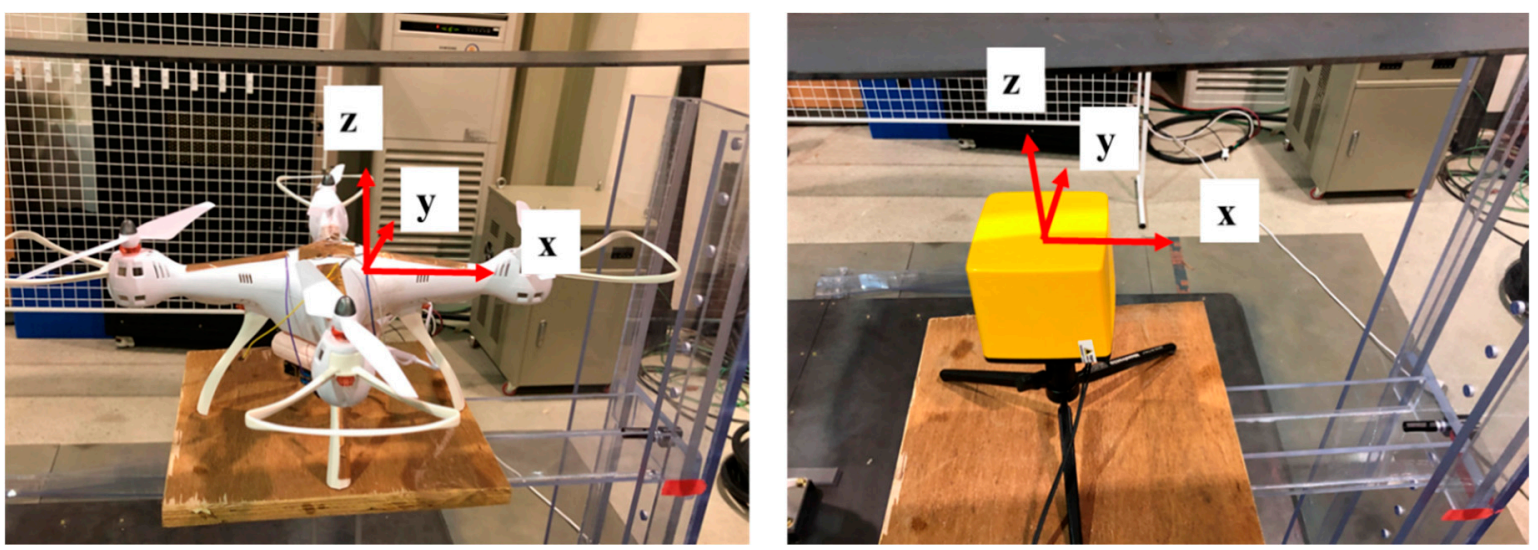

(b)

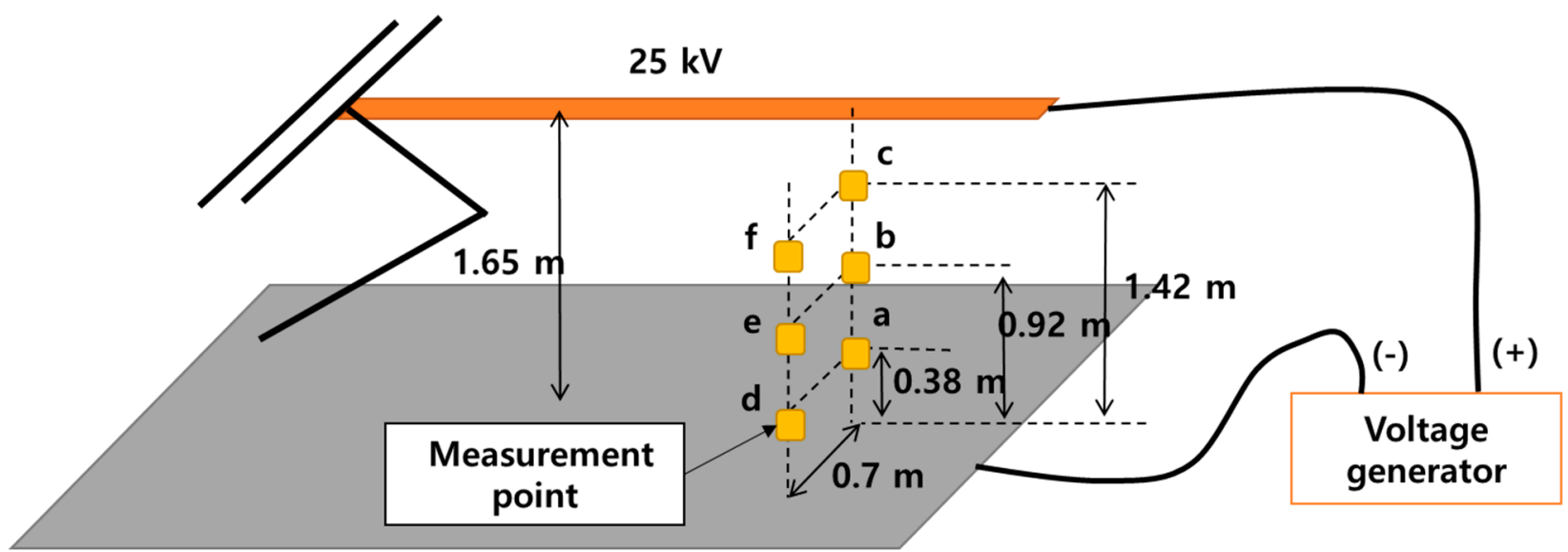

(c)

Figure 6. (a) Schematic of the experimental setup for electric field generation; (b) measurement direction of the sensor; and (c) position of the measurement positions.

To examine the relationship between the proposed sensor and the measured electric field, the measured electric field and sensor voltages were compared with respect to changes in the supply voltage at position $e$, as indicated in Figure 6c. Figure 7 shows the values of 
the measured electric field and the voltage measured by each proposed sensor when the supply voltage of the overhead line was increased from 1 to $25 \mathrm{kV}$. Over the electric field range of 0.5 to $10.1 \mathrm{kV} / \mathrm{m}$, voltages of 0 to $0.77 \mathrm{~V}$ were measured by the proposed sensor. From these measured values, it can be seen that as the voltage is consistently increased, the intensity of the electric field also increases. Accordingly, the values of the voltage measured by the sensor also demonstrated a constant increase.

\section{Measured data}

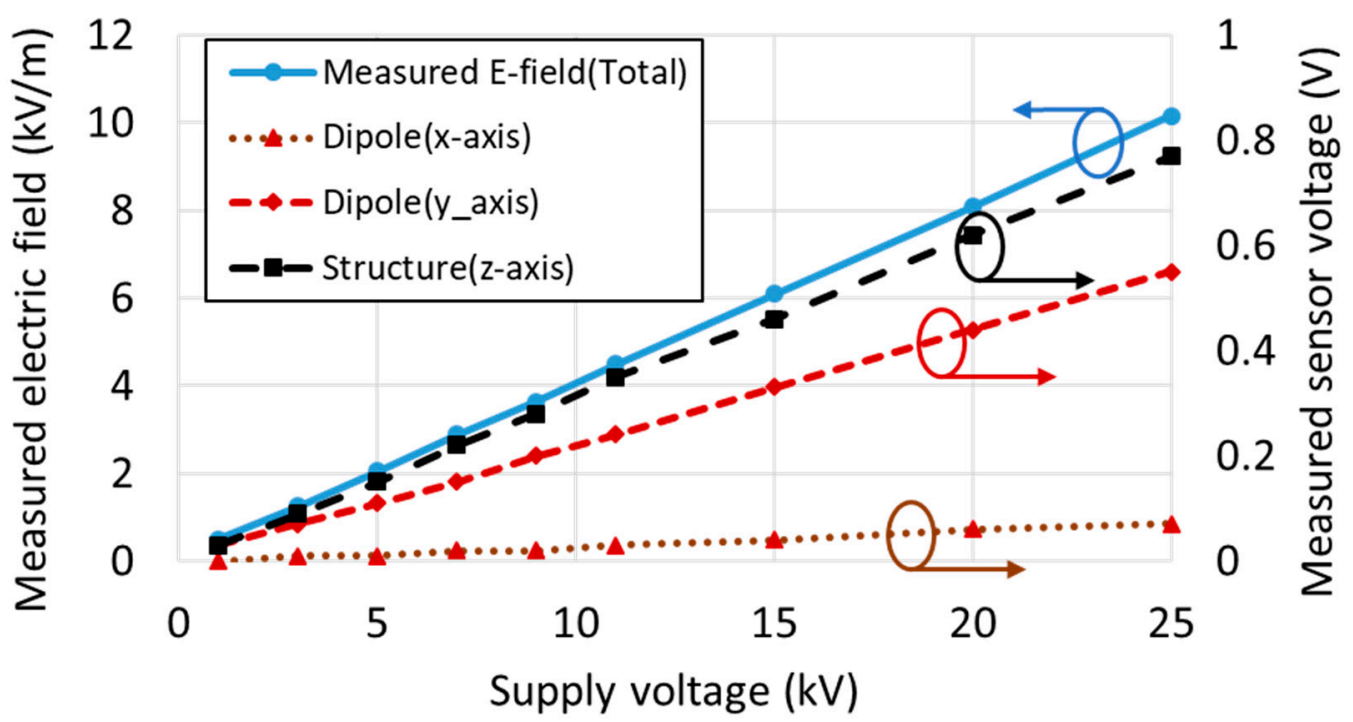

Figure 7. Measured values of the electric field and each axis sensor voltage in the laboratory setting with an increasing voltage applied to the overhead line.

Next, the electric field and sensor voltage were measured from positions $a$ to $f$, as indicated in Figure 6c. The results are presented in Table 4.

Table 4. Comparison of the measured electric field and sensor voltage with respect to the position of the measurement points.

\begin{tabular}{|c|c|c|c|c|c|c|c|c|}
\hline \multirow{2}{*}{ Position } & \multicolumn{4}{|c|}{ Measured Electric Field (kV/m) } & \multicolumn{3}{|c|}{ Measured Sensor Voltage (V) } & \multirow{2}{*}{$\begin{array}{l}\text { Compensated } \\
\text { Sensor Value }\end{array}$} \\
\hline & $x$-Direction & $y$-Direction & z-Direction & Total & Dipole (x) & Dipole (y) & Structure $(\mathrm{z})$ & \\
\hline$a$ & 1.40 & 2.99 & 20.12 & 20.39 & 0.53 & 0.68 & 1.69 & 20.35209 \\
\hline$b$ & 3.70 & 1.68 & 14.09 & 14.66 & 0.26 & 0.50 & 1.20 & 14.11101 \\
\hline$c$ & 1.81 & 3.03 & 30.31 & 30.52 & 0.96 & 0.75 & 2.27 & 28.78331 \\
\hline$d$ & 0.68 & 0.60 & 15.99 & 16.01 & 0.41 & 0.69 & 1.33 & 16.01497 \\
\hline$e$ & 2.46 & 3.84 & 9.08 & 10.16 & 0.07 & 0.55 & 0.77 & 10.16209 \\
\hline$f$ & 2.27 & 6.31 & 2.49 & 7.16 & 0.16 & 0.40 & 0.15 & 7.155126 \\
\hline
\end{tabular}

The proposed sensor has a structure that receives the electric field of each axis. However, since the distance between the ADC and the sensor exists when installing the sensor on the drone, lead wires are needed to electrically connect it. This lead wire also works as a part of the sensor. No matter how well the designed sensor receives only one-direction electric field, if it is manufactured, it can receive an electric field in the other direction. If the magnitude of the electric field measured in each direction is $M x, M y$, and $M z$, and 
the voltage induced from the sensor in each direction is set as $V x, V y, V z$, the relationship between electric field and the induced voltage can be expressed as follows.

$$
\begin{gathered}
M_{x}=a_{11} V_{x}+a_{12} V_{y}+a_{13} V_{z} \\
M_{y}=a_{21} V_{x}+a_{22} V_{y}+a_{23} V_{z} \\
M_{z}=a_{31} V_{x}+a_{32} V_{y}+a_{33} V_{z} \\
\bar{M}=A \bar{V}
\end{gathered}
$$

where the matrix $A$ is weighting matrix. If the coefficient of $A$ is determined, the electric field in each direction can be calculated from the voltage of the sensor. Using this, the magnitude of the total electric field can be calculated from the following equation.

$$
M_{\text {Total }}=\sqrt{M_{x}^{2}+M_{y}^{2}+M_{z}^{2}}
$$

where, we named $M_{\text {Total }}$ as the compensated sensor value.

To obtain the A matrix, the measured sensor values and electric field values from three points $d, e$, and $f$ in Figure $6 \mathrm{c}$ were used. If there are measured values for three different points, $V$ vector and $\boldsymbol{M}$ vector can be expressed in the form of a $3 \times 3$ matrix, the A matrix can be calculated from the following formula.

$$
A=M V^{-1}
$$

The compensated sensor values extracted from the calculated $A$ are shown on the right side of Table 4 . It can be seen that the measured electric field and sensor value at positions $a$ to $f$ show similar trends. For positions $d$ to $f$, which are located at a distance of $0.7 \mathrm{~m}$ in the horizontal direction from positions $a$ to $c$, the values of the electric field are observed to not be significantly affected by the height. The measured electric field and sensor voltage decrease as it approaches point $f$.

Figure 8 shows the results of a simulation of the electric field intensity when a voltage of $25 \mathrm{kV}$ is applied to the laboratory setup. It can be seen that the simulated electric field intensity shows a similar trend to the measured electric field intensity for each position of the sensor. The simulation result has a slightly lower electric field value than the measurement result. Considering the positions at which some discrepancies from the measured values are observed, these differences may have occurred because the simulation was unable to the accurately model the metal apparatus at these locations (i.e., the pantograph, supply power cable, etc.). 


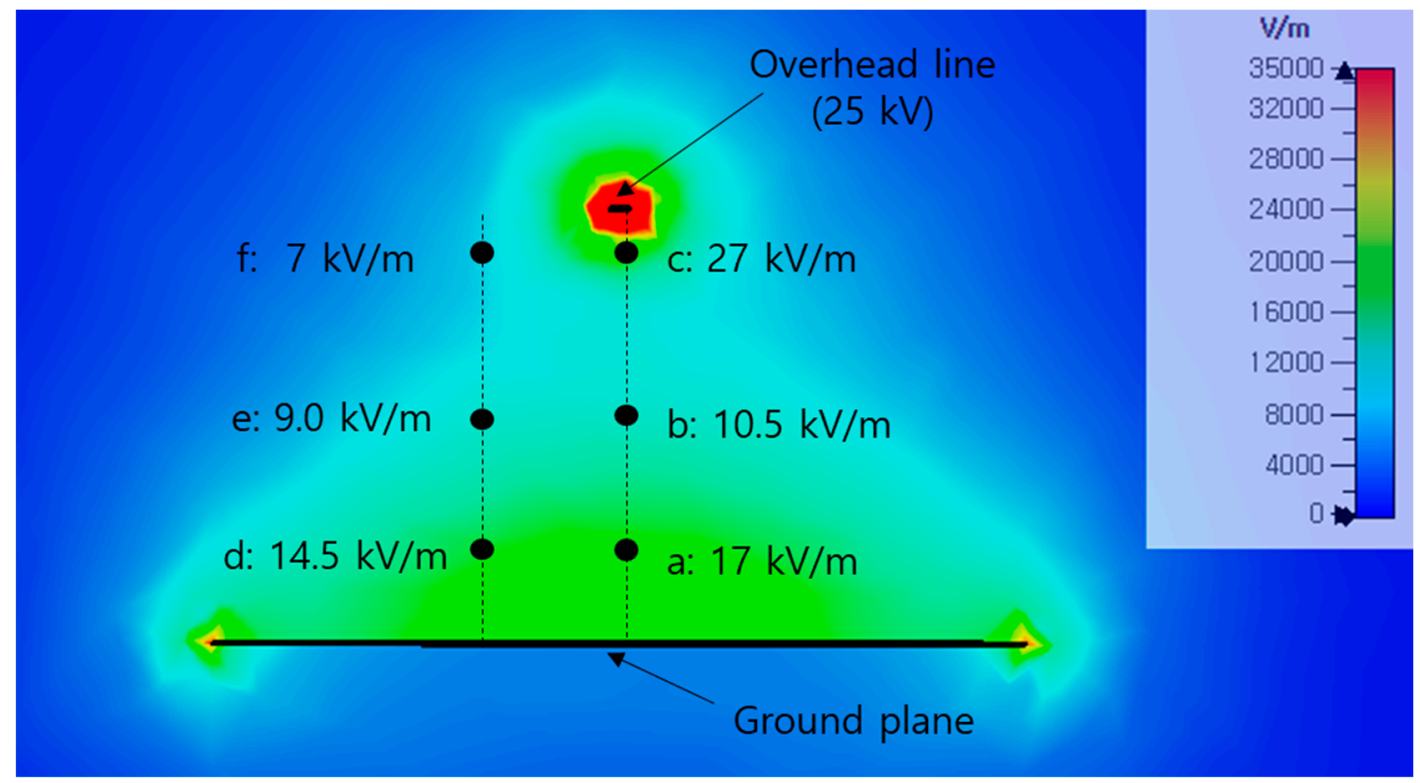

(a)

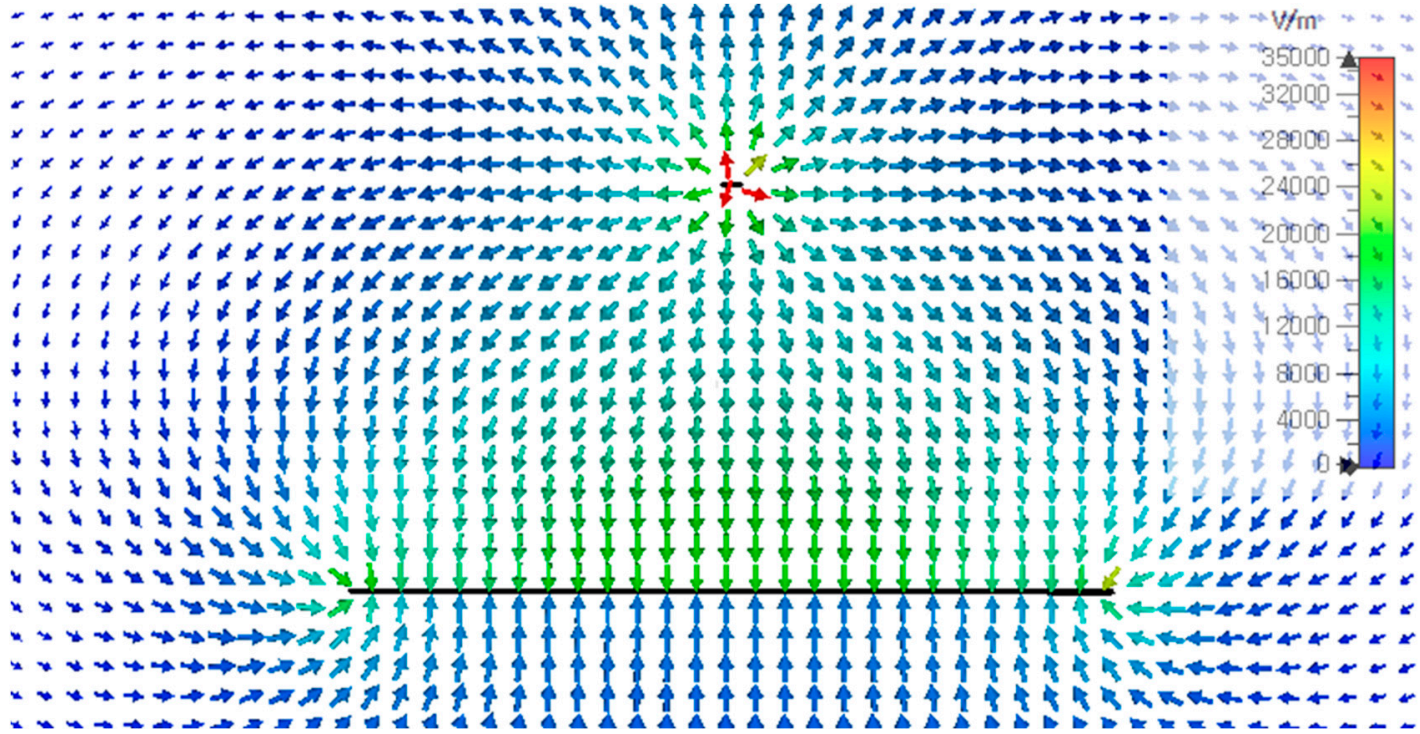

(b)

Figure 8. Distribution of the simulated electric field: (a) contour plot; (b) vector plot.

Figure 9 shows a real-world railway environment used to obtain experimental measurements. The electric field and the voltage of the sensor were measured at three points located $1.5 \mathrm{~m}$ from the center of the railway line. The measurement results are shown in Table 5. The compensated sensor values are slightly higher than the measured electric field. However, the trend does not deviate. In the railway environment, the voltage applied to the overhead line was $25 \mathrm{kV}$, and the distribution of the simulated electric field in this experimental environment is shown in Figure 10. The value of the simulated electric field at point $\mathrm{g} 1$ is $1.8 \mathrm{kV} / \mathrm{m}$, which is lower than the measured value of $4.05 \mathrm{kV} / \mathrm{m}$. In the real-world environment, several overhead lines with an applied voltage in the vicinity of the measurement positions may affect the obtained measurements, thereby resulting in higher values of the measured electric field. 


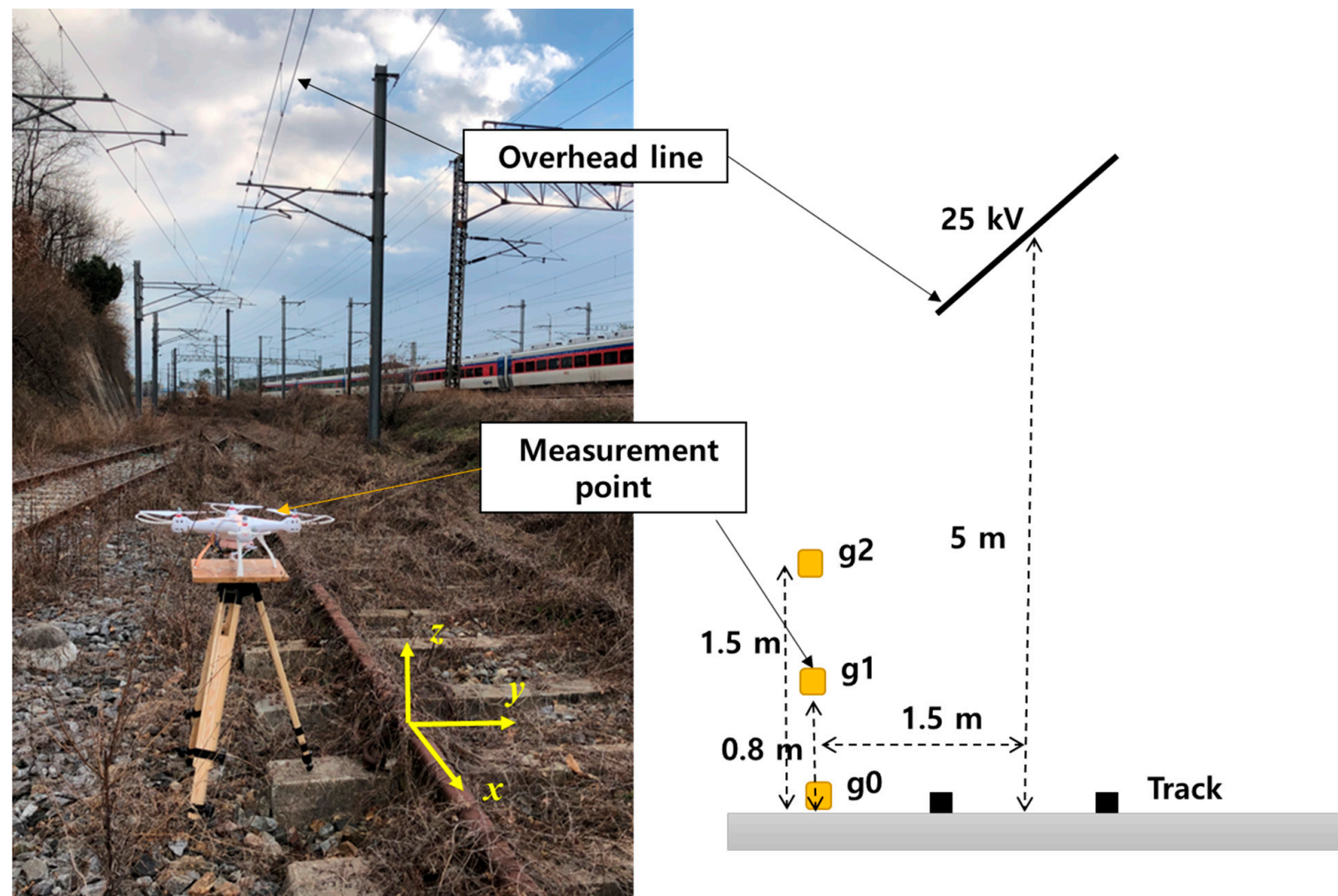

Figure 9. Location of the measurement point of the sensor in a railway environment with overhead lines.

Table 5. Measured values of the electric field and sensor voltage in the railway environment.

\begin{tabular}{|c|c|c|c|c|c|c|c|c|}
\hline \multirow{2}{*}{ Position } & \multicolumn{4}{|c|}{ Measured Electric Field (kV/m) } & \multicolumn{3}{|c|}{ Measured Sensor Voltage (V) } & \multirow{2}{*}{$\begin{array}{l}\text { Compensated } \\
\text { Sensor Value }\end{array}$} \\
\hline & $x$-Direction & $y$-Direction & z-Direction & Total & Dipole (x) & Dipole (y) & Structure $(\mathrm{z})$ & \\
\hline g0 & 0.69 & 0.35 & 2.83 & 2.94 & 0.13 & 0.14 & 0.30 & 3.684274 \\
\hline g1 & 0.71 & 0.68 & 3.93 & 4.05 & 0.20 & 0.17 & 0.36 & 4.540885 \\
\hline g2 & 0.69 & 0.79 & 4.05 & 4.18 & 0.17 & 0.16 & 0.39 & 4.829291 \\
\hline
\end{tabular}

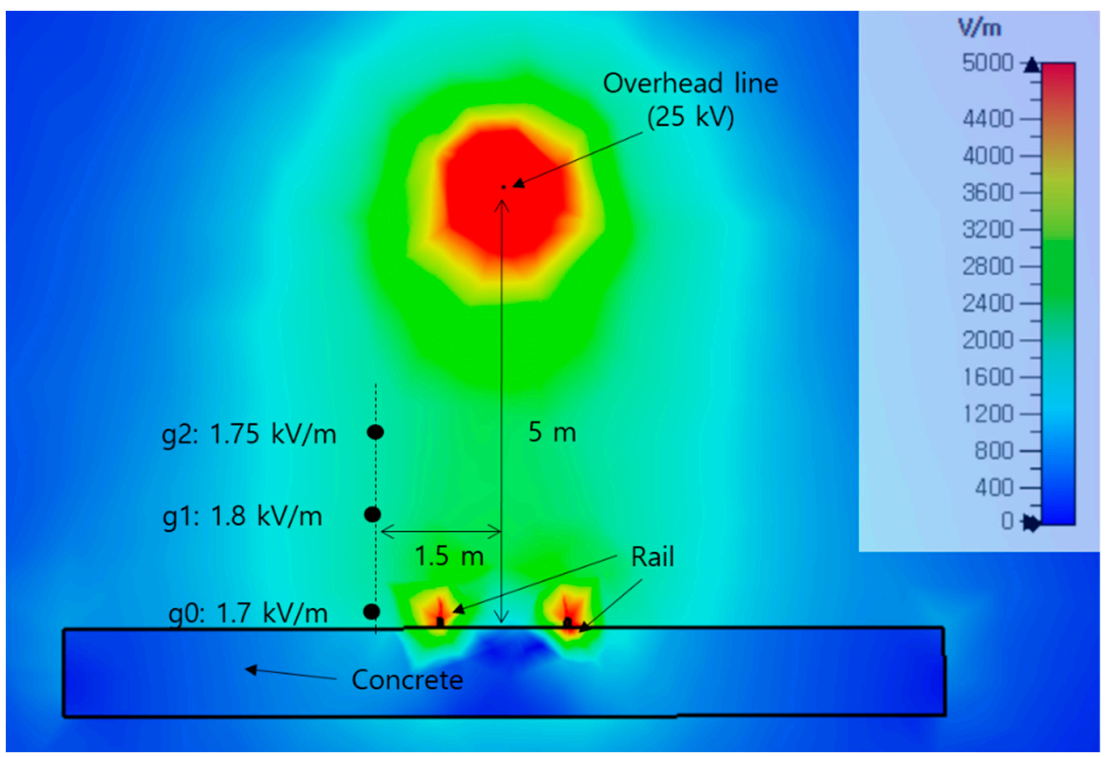

(a)

Figure 10. Cont. 


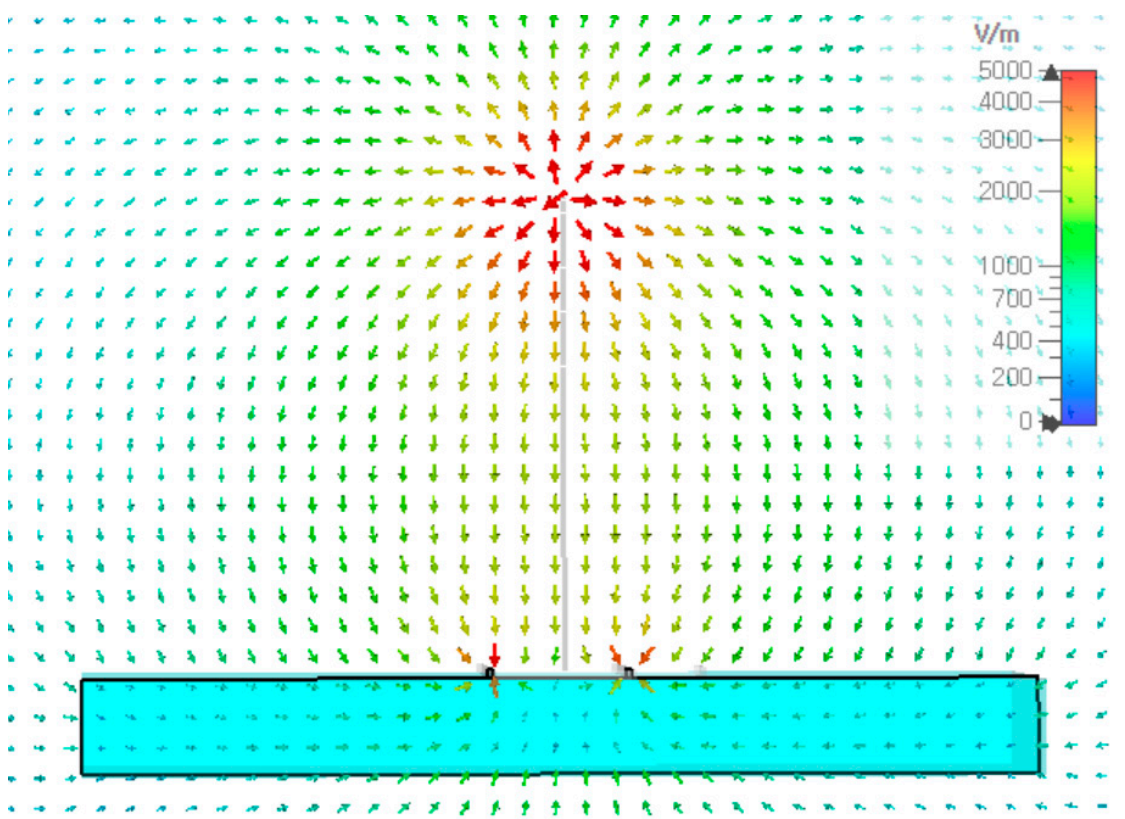

(b)

Figure 10. Simulated electric field distribution in the railway environment: (a) contour plot; (b) vector plot.

From the above results, it can be seen that the proposed sensor produces compensated sensor values that are $7 \mathrm{~V}$ or greater in a large electric field environment. Therefore, if a system is designed such that it provides an indication when the measured sensor value is above a pre-determined value, a drone may be able to detect approaching the high-voltage lines. In this study, the measured voltage of the sensor was made in units of $0.01 \mathrm{~V}$. Because this unit is not affected by ambient noise. If this value is roughly converted to an electric field value, it corresponds to $0.3 \mathrm{kV} / \mathrm{m}$. Therefore, it can be seen that the proposed sensor has the resolution to distinguish an electric field of $0.3 \mathrm{kV} / \mathrm{m}$.

\section{Conclusions}

In this study, a sensor for use in drones to measure electric fields in their surroundings is proposed to prevent contact between drones and high-voltage power lines. Because the voltage measurements achieved by the sensor are determined by the length of the sensor, a method for incorporating the body of the drone as a part of the sensor was proposed. In the structure of the proposed sensor, two dipole antenna structures were used to detect the electric field in the horizontal direction. In addition, to measure the electric field in the vertical direction, a structure using a metal plate and a metal post was proposed.

The voltage induced in the proposed sensor with respect to the electric field was measured in both laboratory and real-world railway environments. In the laboratory environment, the voltage of the proposed sensor was measured to be 0 to $0.77 \mathrm{~V}$ for an electric field in the range of 0.5 to $10.1 \mathrm{kV} / \mathrm{m}$. In the real-world railway environment, the measured electric field was $4.05 \mathrm{kV} / \mathrm{m}$ at a height of $0.8 \mathrm{~m}$, and the compensated sensor value was 4.54 . The sensor proposed in this study may be used such that drones can detect high-voltage power lines through measurements of high electric fields.

Author Contributions: Conceptualization, J.H.K. and G.L.; investigation, G.L. and G.K.; data curation, J.-Y.K.; supervision, J.H.K. All authors have read and agreed to the published version of the manuscript.

Funding: This work was supported by a grant from the R\&D Program of the Korea Railroad Research Institute. 
Institutional Review Board Statement: Not applicable.

Informed Consent Statement: Not applicable.

Data Availability Statement: Not applicable.

Conflicts of Interest: The authors declare no conflict of interest.

\section{References}

1. Cao, W.; Zhu, L.; Han, J.; Wang, T.; Du, Y. High voltage transmission line detection for uav based routing inspection. In Proceedings of the IEEE/ASME International Conference on Advanced Intelligent Mechatronics, Wollongong, NSW, Australia, 9-12 July 2013; pp. 554-558.

2. Gioi, R.G.; Jakubowicz, J.; Morel, J.M.; Randall, G. LSD: A fast line segment detector with a false detection control. IEEE Trans. Patt. Anal. Mach. Intell. 2010, 32, 722-732. [CrossRef] [PubMed]

3. Wang, J.; Yan, X.; Zhong, L.; Zhu, X. Simulation and Test of a Contactless Voltage Measurement Method for Overhead Lines Based on Reconstruction of Integral Node Parameters. Sensors 2020, 20, 246. [CrossRef] [PubMed]

4. Zhou, Q.; He, W.; Li, S.; Hou, X. Research and Experiments on a Unipolar Capacitive Voltage Sensor. Sensors 2015, 15, 20678-20697. [CrossRef] [PubMed]

5. Zhou, Q.; He, W.; Xiao, D.; Li, S.; Zhou, K. Study and Experiment on Non-Contact Voltage Sensor Suitable for Three-Phase Transmission Line. Sensors 2016, 16, 40. [CrossRef] [PubMed]

6. Si, D.; Wang, J.; Wei, G.; Yan, X. Method and Experimental Study of Voltage Measurement Based on Electric Field Integral with Gauss-Legendre Algorithm. IEEE Trans. Instrum. Meas. 2020, 69, 2771-2778. [CrossRef]

7. Misakian, M. ELF electric and magnetic field measurement methods. In Proceedings of the International Symposium on Electromagnetic Compatibility, Dallas, TX, USA, 9-13 August 1993; pp. 150-155.

8. Zolj, A.; Makarov, S.N.; de Lara, L.N.; Nummenmaa, A. Electrically Small Dipole Antenna Probe for Quasistatic Electric Field Measurements in Transcranial Magnetic Stimulation. IEEE Trans. Magn. 2019, 55, 1-10. [CrossRef] [PubMed]

9. Bassen, H.; Smith, G. Electric field probes-A review. IEEE Trans. Antennas Propag. 1983, 31, 710-718. [CrossRef]

10. Smith, G.S. Limitations on the Size of Miniature Electric-Field Probes. IEEE Trans. Microw. Theory Tech. 1984, 32, 594-600. [CrossRef]

11. Liu, Z.; Li, P.; Tian, B.; Zhao, J.; Hu, Y.; Sun, H.; Yin, X.; Wang, Z.; Guo, M. Current and Voltage Measurement Method Based on Magnetic and Electric Field Sensors for Smart Grid Applications. In Proceedings of the 2020 IEEE 4th Conference on Energy Internet and Energy System Integration, Wuhan, China, 30 October-1 November 2020; pp. 2783-2786.

12. Cui, Y.; Yuan, H.W.; Song, X.; Zhao, L.X.; Liu, Y.M.; Lin, L.W. Model, Design, and Testing of Field Mill Sensors for Measuring Electric Fields Under High-Voltage Direct-Current Power Lines. IEEE Trans. Ind. Electron 2018, 65, 608-615. [CrossRef]

13. Kanda, M. Standard probes for electromagnetic field measurements. IEEE Trans. Antennas Propag. 1993, 41, 1349-1364. [CrossRef]

14. Cheng, D. Field and Wave Electromagnetics, 2nd ed.; Addison-Wesley: Reading, MA, USA, 1989; pp. $122-123$. 